IUCM94-015

\title{
Magnetic Oscillations and Quasiparticle Band Structure in the Mixed State of Type-II Superconductors
}

\author{
M.R. Norman \\ Materials Science Division, Argonne National Laboratory, Argonne, IL 60439 \\ A.H. MacDonald \\ Department of Physics, Indiana University, Bloomington, IN 47405 \\ Hiroshi Akera \\ Faculty of Engineering, Hokkaido University, Sapporo 060, Japan
}

(May 9, 2018)

\begin{abstract}
We consider magnetic oscillations due to Landau quantization in the mixed state of type-II superconductors. Our work is based on a previously developed formalism which allows the mean-field gap equations of the Abrikosov state to be conveniently solved in a Landau level representation. We find that the quasiparticle band structure changes qualitatively when the pairing selfenergy becomes comparable to the Landau level separation. For small pairing self-energies, Landau level mixing due to the superconducting order is weak and magnetic oscillations survive in the superconducting state although they are damped. We find that the width of the quasiparticle Landau levels in this regime varies approximately as $\Delta_{0} n_{\mu}^{-1 / 4}$ where $\Delta_{0}$ is proportional to the magnitude of the order parameter and $n_{\mu}$ is the Landau level index at the Fermi
\end{abstract}


energy. For larger pairing self-energies, the lowest energy quasiparticle bands occur in pairs which are nearly equally spaced from each other and evolve with weakening magnetic field toward the bound states of an isolated vortex core. These bands have a weak magnetic field dependence and magnetic oscillations vanish rapidly in this regime. We discuss recent observations of the de Haas-van Alphen effect in the mixed state of several type II superconductors in light of our results.

74.60.-w, 71.25.Hc, 74.25.Jb

Typeset using REVTEX 


\section{INTRODUCTION}

For normal state electrons the study of de Haas-van Alphen (dHvA) oscillations, magnetic oscillations associated with Landau quantization in a magnetic field, has proved to be one of the most revealing probes of low-energy quasiparticle excitations.1 1 In the superconducting state, however, the dHvA effect has not been studied systematically and a great deal of confusion has surrounded its interpretation when the effect has been observed. An external magnetic field is completely screened from the bulk of a superconductor at sufficiently weak magnetic fields; this state of a superconductor is known as the Meissner state. For type-II superconductors a second superconducting state in which the external field is only partially screened, the Abrikosov 2 vortex lattice state or mixed state, occurs in stronger external magnetic fields. It is in this state that magnetic oscillations can occur. The first observation of magnetic oscillations in the Abrikosov state occurred nearly twenty years ago and interest has been renewed recently with their observation in such materials as $\mathrm{NbSe}_{2}$, $\mathrm{V}_{3} \mathrm{Si}$, 期 $\mathrm{Nb}_{3} \mathrm{Sn}, \mathrm{H}$ and in the high temperature superconductors YBCO 80 and BKBO 11

Theoretically it was realized quite early 120 that the upper critical magnetic field, $H_{c 2}$, above which the Abrikosov state gives way to the normal state, shows magnetic oscillations due to Landau quantization. However much less progress has been made on the question of what happens to dHvA oscillations for fields substantially below $H_{c 2}$, principally because of complications introduced by the broken translational symmetry of the vortex lattice state. Several authors have addressed the limit where pairing occurs within a single Landau level resulting in important simplifications.1721 This limit can pertain at low temperatures to fields just above the semiclassical $H_{c 2}$ where the order parameter is very small. (Plausible suggestions have been made 22 concerning the possibility of reentrant superconductivity for $H>>H_{c 2}(T=0)$ but these still lack experimental verification and considerations of the vortex lattice state in the reentrant regime 17 have been largely didactic.) The works of Maki23 and of Stephen,24 whose analyses are based on semiclassical approximations for the

electron Greens function 25 in the mixed state, are more relevant to the intermediate field 
situation. Their results are based in part on different versions of an approximation which involves positional averaging over the vortex lattice and was first introduced by Brandt et al.25 In this approach the vortex lattice acts much like a random scattering potential which contributes to the inverse lifetime of quasiparticle states without shifting them away from the Fermi level. When Landau quantization is accounted for this scattering broadens the Landau levels and therefore reduces the amplitude of the dHvA oscillations. An interesting alternate approach was taken in a paper by Maniv et al, 16 which studies magnetic oscillations in the superconducting condensation energy.

In this paper we study magnetic oscillations in the mixed state using a previously developed formalism 26 which allows the electronic structure of the Abrikosov lattice state to be calculated conveniently in a Landau level basis. This approach treats the effects of Landau quantization without approximation within mean-field theory. (A similar formalism was independently derived by Rajagopal.27) Our conclusions are based in part on numerical calculations for representative models of weak coupling superconductors and, in part, on analytic perturbative calculations. Our results differ qualitatively from those obtained in earlier work. We find that magnetic oscillations in the normal state free-energy and in the superconducting condensation energy have a strong tendency to cancel, making studies of the condensation energy alone misleading. (The condensation energy is defined as the difference between the free energies of normal and superconducting states.) Close to $H_{c 2}$, the off-diagonal self-energy of the mean-field equations is small, and we find in agreement with Maki23 and Stephenes that the quasiparticle bands consist of broadened Landau levels which are not substantially shifted away from the Fermi level. However we find that the width of the Landau levels varies with the magnitude $\left(\Delta_{0}\right)$ of the order parameter and the Landau level index at the Fermi level $\left(n_{\mu}\right)$ as $\Delta_{0} n_{\mu}^{-1 / 4}$ rather than as $\Delta_{0}^{2} n_{\mu}^{-1 / 2}$. This qualitative difference is important for the interpretation of experiments which show magnetic oscillations in the mixed state and is a consequence of the degeneracy of the Landau bands in the absence of superconducting order. When the width of the Landau quasiparticle bands becomes comparable to the Landau level separation we find that there is a crossover in the 
quasiparticle spectrum. The lowest energy quasiparticle bands evolve into tight-binding bands corresponding to the bound states of isolated vortices and are insensitive to magnetic field strength. The higher energy quasiparticle bands retain Landau level character but are shifted well away from the Fermi level. Magnetic oscillations are negligible in this regime.

Our paper is organized as follows. In Section प⿴囗十 we briefly review our formalism for solving the non-linear gap equations for the Abrikosov state in a Landau level representation. Previous calculations using this formalism focused on the self-consistently calculated

order parameter 26 and the tunneling spectra.28 Some practical details of these calculations are discussed in Section III. In Section IV we focus on the quasiparticle electronic structure. The non-linear gap equations are solved numerically for a representative model of a weak-coupling superconductor. dHvA oscillations are a consequence of the dependence of the electronic structure on magnetic field, and specifically result from Landau levels crossing through the chemical potential as a function of magnetic field. In Section $\nabla$ we discuss in detail the consequences for magnetic oscillations of our results for the quasiparticle spectrum in the mixed state. Functional fits to the suppression of the magnetic oscillations are derived and related to experimental data. Section $\nabla]$ contains a discussion of the magnetic field dependence of the energy from a variational point of view which aims to explain the importance of competition between pairing energy and kinetic energy in reducing the amplitude of magnetic oscillations. Finally in Section VII we briefly summarize our results.

\section{NON-LINEAR GAP EQUATIONS IN THE ABRIKOSOV LATTICE STATE}

The discussion in this section and the illustrative calculations in subsequent sections are for the case of two-dimensional superconductors in perpendicular magnetic fields. The restriction to two dimensions in this section is simply a matter of notational simplicity; in the models we use, the quasiparticle states factorize into a planar part which is explicitly exhibited below and a part associated with the degree of freedom along the field direction. Along the field direction the pairing occurs between time-reversed partners, $k_{z}$ and $-k_{z}$, as 
at zero magnetic field; including the third direction necessitates merely the addition of a new index for the quasiparticle states which must be summed over in constructing the selfconsistent off-diagonal self-energy. The strength of the magnetic oscillations are stronger in the two-dimensional case than they would be if we chose a three-dimensional model; in subsequent sections, we have added comments at those points where the translation to the three-dimensional case is not obvious. The use of a two-dimensional model to illustrate the points we wish to make substantially simplifies our numerical calculations. The threedimensional case is straightforward to treat, but is more computationally demanding due to (i) having to treat a larger number of Landau parabolas as opposed to discrete Landau levels and (ii) having to work with a larger number of $\vec{k}$ points in a 3D wavevector space as opposed to a $2 \mathrm{D}$ one.

The effect of a magnetic field on electronic orbitals in quantum mechanics appears through the vector potential $(\nabla \times \vec{A}=\vec{B})$. For a non-zero average magnetic field $\vec{A}$ is $\sim L$ where $L$ is the system size and, unless the electronic states are localized, even a weak magnetic field cannot be treated perturbatively. A quantum treatment of the electronic structure of the Abrikosov lattice state must be performed in a basis where the average magnetic field $\left(B_{0}\right)$ is accounted for exactly; it is this requirement which fundamentally changes the nature of the mean-field equations. To describe the vortex lattice state it is convenient to use a Landau gauge $\left(\vec{A}=\left(0, B_{0} x, 0\right)\right)$ in which the kinetic energy eigenstates are

$$
\phi_{N X}(\vec{r})=\frac{1}{\sqrt{L_{y}}} e^{-i X y / l^{2}} \phi_{N}\left(\frac{x-X}{l}\right)
$$

where $\phi_{N}(x)=\left(2^{N} N ! \sqrt{\pi} l\right)^{-1 / 2} e^{-x^{2} / 2} H_{N}(x)$ and $H_{N}$ is a Hermite polynomial of order $\mathrm{N}$ $\left(\ell^{2}=\hbar c / e B\right)$. The non-linear gap equations for non-uniform superconductors, 29 commonly known as the Bogoliubov-de Gennes (BdG) equations, are obtained by making a generalized Hartree-Fock factorization of the equation of motion for electron creation and annihilation operators. To exploit the translational symmetry of the vortex lattice state we first form magnetic Bloch states from the $\phi_{N X}$ : 


$$
\phi_{N \vec{k}}=\sqrt{\frac{a_{x}}{L_{x}}} \sum_{t} e^{i k_{x} a_{x} t} e^{i \pi t^{2} / 4} \phi_{N,-k_{y} l^{2}+t a_{x}}(\vec{r}) .
$$

where the sum is over all integers, and for general $a_{x}$ and $a_{y}$, one restricts $k_{y}$ to an interval of length $a_{x} / \ell^{2}$ and $k_{x}$ to an interval of length $2 \pi / a_{x}$. (Note that there is one wavevector for each state in the Landau level.) This basis is convenient when solving the gap equations for a vortex lattice with primitive lattice vectors $\left(0, a_{y}\right)$ and $\left(a_{x},-a_{y} / 2\right)$ where $a_{x} a_{y}=\pi / \ell^{2}$. Solutions corresponding to various vortex lattices can be found by choosing different values for the vortex lattice aspect ratio $R=2 a_{x} / a_{y}$ with $R=1$ for a square lattice and $R=\sqrt{3}$ for the triangular lattice, which is the ground state when Ginzburg-Landau theory applies. We see below that in this representation only states with opposing Bloch wavevectors are coupled by the off-diagonal self-energy, as in the zero-field mean-field equations. Unlike the zero-field case, however, the basis orbitals are labeled by wavevector and by a Landau level index and the pairing self-energy is not diagonal in the Landau level index. The BdG secular matrix for the case of singlet pairing is of dimension $2 N_{L}$ where $N_{L}$ is the number of Landau levels involved in the pairing:26

$$
\begin{gathered}
\left(\xi_{N}-E_{\vec{k}}^{\mu}\right) u_{N \vec{k}}^{\mu}+\sum_{M} F_{\vec{k} N M} v_{M \vec{k}}^{\mu}=0 \\
\left(-\xi_{N}-E_{\vec{k}}^{\mu}\right) v_{N \vec{k}}^{\mu}+\sum_{M} F_{\vec{k} M N}^{*} u_{M \vec{k}}^{\mu}=0 .
\end{gathered}
$$

Here $u_{N \vec{k}}^{\mu}$ is the coefficient of $\phi_{N \vec{k}}$ and $v_{N \vec{k}}^{\mu}$ is the coefficient of $\phi_{N-\vec{k}}^{*}$ in the real-space Bogoliubov amplitudes $u(\vec{r})$ and $v(\vec{r})$ for the $\mu^{\prime}$ th solution, and $\xi_{N}=\hbar \omega_{c}(N+1 / 2)-\mu$ is the kinetic energy of the $N$ - th Landau level measured from the chemical potential. $\left(\omega_{c}=e B / m c\right.$ is the cyclotron frequency.) $F_{\vec{k} N M}$ is the $\vec{k}$ dependent pairing self-energy matrix in the Landau level representation and must be determined self-consistently as we discuss below.

In deriving these equations we have assumed that the magnetic flux density $(B)$ in the superconductor is uniform. For a strong type II superconductor, this approximation should be accurate except for external fields close to the lower critical field. The above equations also assume that we are dealing with an electron gas model which is translationally invariant in 
the normal state; the discussion could be modified to include band structure effects but that complication is not discussed here. As mentioned above, Eqs. [3, 1 , have been written in a form appropriate to the two-dimensional case to avoid unnecessarily cluttering the notation. The illustrative calculations performed in subsequent sections are for a two-dimensional electron gas model with attractive interactions, since aspects associated with the band structure of a particular material are not important for the issues we plan to address and since magnetic oscillation phenomena are more pronounced in the two-dimensional case.

We use a simple Bardeen-Cooper-Schrieffer (BCS) model for the frequency dependence of the attractive interaction giving rise to the pairing self-energy so that the sums over Landau levels are restricted to states whose kinetic energies lie within a cutoff energy $\left(\omega_{D}\right)$ of the chemical potential. For a $\delta$-function attractive interaction the off-diagonal self-energies are given by 26

$$
F_{\vec{k} N M}=\frac{-\lambda \hbar \omega_{c}}{2} \sum_{j} \chi_{M+N-j}(\vec{k}) D_{j}^{M N} \Delta_{j}
$$

with

$$
\begin{gathered}
\chi_{j}(\vec{k})=\sum_{t} e^{i 2 k_{x} a_{x} t} e^{-i \pi t^{2} / 2} \chi_{j}\left(2 k_{y} l^{2}+2 t a_{x}\right) \\
\chi_{j}(Y)=\left(\frac{l}{\sqrt{2}}\right)^{1 / 2} \phi_{j}\left(\frac{Y}{\sqrt{2} l}\right)
\end{gathered}
$$

and

$$
D_{j}^{N M}=\left(\frac{j !(N+M-j) ! N ! M !}{2^{N+M}}\right)^{1 / 2} \sum_{m=0}^{j} \frac{(-1)^{N-m}}{(j-m) !(N+m-j) !(M-m) ! m !}
$$

In these equations $\lambda$ is the BCS coupling constant $\left(\lambda \hbar \omega_{c}=V /\left(2 \pi \ell^{2}\right)\right.$ where $\mathrm{V}$ is the strength of the attractive interaction). The sum over $j$ in Eq. [5] is over the possible partitionings of the total quantized kinetic energy of the pair, $\hbar \omega_{c}(N+M+1)$, into contributions from the pair center of mass motion, $\hbar \omega_{c}(j+1 / 2)$, and the pair relative motion, $\hbar \omega_{c}(N+M-j+1 / 2)$.

The derivation of this form for the pairing self-energy closely follows the derivation 13 of the linearized gap equations which is greatly simplified by making unitary transformations 
for two-particle states between the representations in which each particle has a definite Landau quantized kinetic energy and the representation in which definite Landau quantized kinetic energies reside in the center of mass and relative motion of the pairs. The $D_{j}^{N M}$, for which explicit expressions are given in Eq. [8], are the matrix elements of the unitary transformation. $\left(D_{j}^{N M}\right)^{2}$ is the probability that a pair of electrons in Landau levels $N$ and $M$ will have center-of-mass kinetic energy $\hbar \omega_{c}(j+1 / 2) . \Delta_{j}$ in Eq. [5] is the amplitude for electron pairing with center-of-mass kinetic energy $\hbar \omega_{c}(j+1 / 2)$ and is directly related to the Landau level expansion of the Ginzburg-Landau order parameter.29.30 In our formalism, the self-consistent solution of the BdG equations reduces to the self-consistent determination of these parameters. In practice it is only necessary to determine a small number of parameters. This is particularly true near $H_{c 2}$ where Ginzburg-Landau theory tells us that only $\Delta_{0}$ (the Abrikosov solution) is significantly different from zero and we will discuss the approximation where only this single parameter is retained at length in Section VI. Even at weaker magnetic fields, it follows from symmetry considerations that for a triangular Abrikosov lattice, $\Delta_{j}$ is different from zero only if $j$ is a multiple of six and this property helps to keep the number of parameters which need to be determined self-consistently small. The higher $j$ components of $\Delta_{j}$ are essential, however, in describing the reduction of the vortex core size compared to the lattice constant of the vortex lattice as the field is reduced. $\Delta_{j}$ is given in terms of the eigenvalues and eigenvectors of the Landau representation BdG equations by the following equation:26

$$
\Delta_{j}=-\sum_{N M} D_{j}^{M N} \sum_{\vec{k}} \frac{4 \pi l a_{x}}{L_{x} L_{y}} \chi_{M+N-j}^{*}(\vec{k}) \sum_{\mu}\left(1-f_{\vec{k} \uparrow}^{\mu}-f_{\vec{k} \downarrow}^{\mu}\right) u_{N \vec{k}}^{\mu} v_{M \vec{k}}^{* \mu}
$$

where $E_{\vec{k}}^{\mu}$ is the $\mu^{\prime}$ th positive eigenvalue of the Landau representation BdG equations, and $f_{\vec{k} \sigma}^{\mu}$ is the Fermi function evaluated at the appropriate quasiparticle energy. If Zeeman coupling is included the Fermi functions should be evaluated at energies,

$$
E_{\vec{k} \sigma}^{\mu}=E_{\vec{k}}^{\mu}-g^{*} \hbar \omega_{c} \sigma / 4
$$

where $\sigma= \pm 1$ is the spin index and $g^{*}$ the effective g-factor $\left(\mathrm{gm}^{*} / \mathrm{m}\right)$. 
The diagonal order parameter in a position representation is related to the $\Delta_{j}$ by

$$
\Delta(\vec{r})=\frac{\left(\sqrt{2} L_{y} l\right)^{1 / 2}}{4 \pi l^{2}} \sum_{j}(-1)^{j} \Delta_{j} \sum_{t} e^{i \pi t^{2} / 2} \phi_{j, \sqrt{2} t a_{x}}(\sqrt{2} \vec{r})
$$

Once the BdG equations have been evaluated self-consistently at a particular magnetic field, the free energy can be calculated using 26

$$
F=\sum_{N \sigma} \xi_{N \sigma} N_{N \sigma}+E_{P}-T S
$$

where the pairing self-energy is

$$
E_{P}=-\lambda \hbar \omega_{c} \frac{L_{x} L_{y}}{8 a_{x} \pi l} \sum_{j}\left|\Delta_{j}\right|^{2}
$$

Here $N_{N \sigma}$ is the occupation number of the $N \sigma$ Landau level

$$
N_{N \sigma}=\sum_{\mu \vec{k}} f_{\vec{k} \sigma}^{\mu}\left|u_{N \vec{k}}^{\mu}\right|^{2}+\left(1-f_{\vec{k} \vec{\sigma}}^{\mu}\right)\left|v_{N \vec{k}}^{\mu}\right|^{2}
$$

and $S$ is the entropy

$$
S=-k_{B} \sum_{\mu \vec{k} \sigma}\left(1-f_{\vec{k} \sigma}^{\mu}\right) \ln \left(1-f_{\vec{k} \sigma}^{\mu}\right)+f_{\vec{k} \sigma}^{\mu} \ln f_{\vec{k} \sigma}^{\mu}
$$

\section{MODEL CALCULATIONS}

In this section we discuss some practical details of the model calculations performed using the above formalism and present some results for the magnetic field dependence of $T_{c 2}$ and the zero-temperature order parameter. This results serve as a characterization of the model for which we will study magnetic oscillations.

We have found that it is necessary to take some care in cutting off the attractive interaction of the model away from the Fermi energy. For many purposes it is adequate to treat the cut-off in the BCS fashion, that is to simply set the attractive interaction to zero when the difference between the normal state quasiparticle energy and the chemical potential exceeds some value. This procedure can lead to undesirable consequences. In our own 
work on the linearized gap equation 13 we found that that it leads to unphysical features in $H_{c 2}$ associated with Landau levels passing through the cut-off energy. In the work of Markiewicz et al, 11 strong features were found in the gap function of the same origin. In our preliminary work on this problem, we found a more severe problem. In the two-dimensional sharp cut-off model with fixed chemical potential, we found that the superconducting condensation energy increases smoothly with increasing field as the Landau level degeneracy increases and then decreases discontinuously as the the number of Landau levels within the pairing cut-off decreases by one. This causes the typical magnetization to be paramagnetic (since the critical temperature increases with field 32 ) with sharp diamagnetic spikes at particular fields. By going to a smooth cut-off, though, we recover the continuous diamagnetic behavior we expect to be caused by the reduction of superconducting condensation energy in a magnetic field. A pragmatic solution is achieved by using a model where the pairing interaction between Landau levels $N$ and $M$ is scaled by $\sqrt{W_{N} W_{M}}$ where

$$
W_{N}=1.55 e^{-\left(\xi_{N} / 0.5 \omega_{D}\right)^{4}}
$$

The sums are still restricted to energies within $\omega_{D}$ of the chemical potential but the interactions at the 'edge' of the pairing window are sufficiently weak that they do not give rise to significant anomalies. We have used this procedure in all our calculations.

We note that because we are using full quantum wavefunctions for the Landau levels, we are currently limited to single particle Landau indices of 60 or less. This practical limit is reached because of numerical difficulties originating in the rapid oscillations of the wavefunctions for large Landau index. This problem could be circumvented by using semiclassical approximations when the Landau level indices are too large.

We have chosen to work in the grand-canonical ensemble where the chemical potential rather than the particle number is held fixed. The difference between the dependence of the magnetization on density and the dependence of the magnetization on chemical potential has been extensively discussed by Shoenberg 33 for the case of the normal state.

For every model we have checked we find that when the external magnetic field is weaker 
than the semiclassical upper critical field, the energy is minimized by a triangular vortex lattice in agreement with Ginzburg-Landau theory. (This is not the case for solutions of the mean-field equations at stronger external fields which are in the reentrant quantum regime.26.28) All the model calculations in subsequent sections of this paper are for triangular flux lattices and one can exploit the symmetries of that situation. It is possible to show that for a triangular vortex lattice the BdG equations have the symmetry of a triangular Brillouin zone with an origin at $\Gamma \equiv\left(\frac{\pi}{4 a_{x}}, \frac{\pi}{2 a_{y}}\right)$. This allows us to limit our sums over wavevectors to $\vec{k}$ 's in an irreducible triangle of the zone with vertices at $\Gamma, M \equiv\left(\frac{3 \pi}{4 a_{x}}, \frac{\pi}{2 a_{y}}\right)$ and $K \equiv\left(\frac{3 \pi}{4 a_{x}}, \frac{\pi}{6 a_{y}}\right)$. The area of this irreducible triangle is $1 / 12$ of the area of the full zone and the labeling of the points at the vertices is the conventional one for symmetry points in a triangular lattice. For most of our calculations, we have used a $\vec{k}$ mesh with a spacing which is 0.2 times the $\Gamma-M$ distance for the wavevector sums, although we have done a number of calculations with a mesh which is twice as fine. The two meshes lead respectively to 21 and $66 \vec{k}$ 's in the irreducible triangle.

Most of the self-consistent calculations in subsequent sections used a coupling constant, $\lambda=0.75$. This choice places the semiclassical upper critical field at a low enough Landau index (strong enough magnetic field) so that we could access most of the semiclassical phase region with our code's restriction on the maximum Landau level index. A large ratio of the cut-off, $\omega_{D}$, to the chemical potential, $\mu$, of $\frac{1}{2}$ was chosen so as to maximize the number of Landau levels involved in the pairing. For the sake of definiteness we choose the g-factor to be zero in our model calculations. (Zeeman spin-splitting does modulate dHvA oscillations in the normal state in a way which is well understood and will have a similar effect in the superconducting state.)

In Fig. 1, we show a plot of the critical temperature, $T_{c 2}$, versus field, for this model. The field is parameterized in terms of $n_{\mu} \equiv \mu / \hbar \omega_{c}-1 / 2$ which gives the Landau level index of the Fermi level. (Note that, in contrast to our previous work, 13 no magnetic oscillations are visible in this curve at larger values of $n_{\mu}$; the oscillations at large $n_{\mu}$ found previously are an artifact of using a sharp cut-off.) At strong enough fields, the critical temperature is 
driven to a low enough value that $k_{B} T_{c 2}$ is much smaller than $\hbar \omega_{c}$ and the system crosses over to the quantum regime where magnetic oscillations in $T_{c 2}$ appear. In this regime a significant portion of the pairing occurs within the Landau level at the Fermi level, even at $T_{c 2}$. These quantum oscillations in $T_{c 2}$ are much less robust in three-dimensional models and do not appear until $k_{B} T_{c 2}<\hbar \omega_{c} / n_{\mu}$. Zeeman spin-splitting and broadening of Landau levels due to disorder also will suppress these oscillations. 1 The maxima in $T_{c 2}$ occur when the density of states at the chemical potential is largest; i.e., when a Landau level occurs at the chemical potential. Regions where $T_{c 2}$ vanishes occur because we work at fixed chemical potential; at fixed particle number, the chemical potential is locked to a Landau level, so cusps occur instead of gaps. For the present model once $n_{\mu}>12$ no quantum effects can be seen in the dependence of $T_{c 2}$ on field.

Also shown in Fig. 1 is a plot of the root-mean-square spatial average of the off-diagonal self-energy in a coordinate representation $\left(F \simeq 0.44 \lambda \hbar \omega_{c} \Delta_{0}\right)$ for this model obtained by solving the non-linear gap equation self-consistently for the same model at $k_{B} T / \mu=10^{-3}$. As we approach the weak-field limit we expect that $\Delta(\vec{r})$ should be constant over much of the area of the system and that $F$ should approach the zero-field BCS energy gap for this model. We see in Fig. 1 that at weaker fields the ratio of $F$ to $T_{c 2}$ approaches a constant. The ratio of these quantities varies from 1.87 for $n_{\mu}=20$ to 1.77 for $n_{\mu}=40$, compared to the zero-field BCS value of 1.76. Magnetic oscillations occur in this curve since the temperature used is low. Their suppression at weaker magnetic fields must originate from changes in the electronic structure associated with superconductivity. It is this suppression which is our primary interest in the present paper.

In Fig. 2, we show the spatial dependence of the self-consistent order parameter at $k_{B} T / \mu=10^{-3}$ along the line connecting neighboring vortex cores. Results are shown for various fields from $n_{\mu}=12$ to $n_{\mu}=39$. At small values of $n_{\mu}$ (i.e. near $H_{c 2}$ ) the order parameter is close to the Abrikosov order parameter which is obtained when only $\Delta_{0} \neq 0$. At larger values of $n_{\mu}$, the vortex core size becomes small compared to the distance between vortex cores and the magnitude of the order parameter becomes nearly constant outside the 
cores as expected. (Many $\Delta_{j}$ need to be non-zero to obtain this behavior.) Interestingly, the dependence on $r$ has oscillations which, as we will see later, are related to discrete quasi-bound states in the vortex cores.

\section{FLUX LATTICE QUASIPARTICLE BANDS}

In this section we look in detail at the quasiparticle band structure in the mixed state. In Figs. 3 and 4 , we show plots of $E(\vec{k})$ along high symmetry directions in the irreducible triangle of the Brillouin zone for the ten lowest energy quasiparticle bands at a field close to the semiclassical $H_{c 2}(T=0)\left(n_{\mu}=15\right)$ and a much weaker magnetic field $\left(n_{\mu}=40\right)$. From Fig. 3 it is clear that the quasiparticle bands at $n_{\mu}=15$ can be regarded as broadened Landau level bands. The quasiparticle bands in the normal state are dispersionless and when $n_{\mu}$ is an integer one band exists at zero energy and two bands at each multiple of $\hbar \omega_{c}$ (particle, hole). dHvA oscillations are appreciable when the band at the Fermi energy is broadened by less than $\hbar \omega_{c}$ and we see in Fig. 3 that this condition is still satisfied at $n_{\mu}=15$. Moreover, zero energy quasiparticles, which contribute strongly to the magnetic oscillations, occur at several $\vec{k}$ points in the zone as can be seen in Fig. 3.

As $n_{\mu}$ increases and the order parameter gets larger the quasiparticle bands continue to broaden, but then cross over to a regime where the lowest energy bands shift away from zero and begin to narrow. In this regime, the eigenvalues have a weak dependence on field. This can be seen in Fig. 5 where the average energy of each pair of bands is plotted in chemical potential units versus $n_{\mu}$. At low $n_{\mu}$, the decrease of the eigenvalues in field is just a reflection of the decrease in cyclotron frequency with decreasing field (i.e., the levels behave like Landau levels). These levels then cross over to field independent behavior at high $n_{\mu}$ with an energy scale characteristic of vortex core bound states. In fact, the low energy quasiparticle bands shown in Fig. 4 occur in pairs and the energies of the lower energy member of the three lowest pair of bands at the $\Gamma$ point are approximately in the ratio of 1:3:5. This is the ratio of energies expected for the lowest energy quasiparticle states bound 
to an isolated vortex core and in fact the lowest energy eigenvalue is close to the predicted 34 value of $\Delta^{2} / \mu$. We believe that the low energy bands seen in Fig. 4 correspond to quasibound quasiparticles which can tunnel from core to core, with a weak field dependence of the energies since the cores have a size much smaller than a magnetic length (see Fig. 2). There are two bands for each bound state since each band has one state for each electron magnetic flux quantum through the system $\left(\Phi_{e}=h c / e\right)$ while the system has one vortex for each superconducting magnetic flux quantum $\left(\Phi_{s c}=\Phi_{e} / 2\right)$, i.e, there are two vortices for each single particle orbital in the normal state Landau levels. There is also a connection of this doubling behavior to the normal state where pairs of excitations (particle, hole) exist, in that if one member of a pair has weight in Landau level $\mathrm{n}$ for the $u$ component of the wavefunction, the other has approximately the same weight in level n-1 for the $v$ component. The difference is that at low $n_{\mu}$, the weight is primarily in one Landau level whereas at high $n_{\mu}$, the weight is distributed among many Landau levels.

This vortex core interpretation of the low energy bands at $n_{\mu}=40$ has been verified by further analyzing the corresponding wavefunctions. Near an isolated vortex core, 34 the quasiparticle amplitudes can be expanded in a set of basis wavefunctions with definite angular momentum. The lowest energy bound quasiparticle state is formed from pairs with angular momentum 0 for the $u$ component and angular momentum -1 for the $v$ component; the next bound quasiparticle state is formed from pairs with angular momentum -1 for the $u$ component and -2 for the $v$ component and so on. The order parameter is proportional to the product $u v^{*}$. The contribution of the $m$-th bound state to the order parameter is therefore proportional to $r^{2 m+1} \exp (i \phi)$ at small $r$ where $r$ and $\phi$ are circular coordinates in the system centered on the vortex core. The common angular dependence is required to selfconsistently maintain the unit vorticity of the vortex. In Fig. 6, we plot the contributions to the order parameter from the lowest two quasiparticle bands for $n_{\mu}=40$. We see that the contribution from the lowest pair of bands is proportional to $r$ at small $r$ while that from the second pair is proportional to $r^{3}$, as expected from the above discussion. Near the vortex core the order parameter is dominated by the contribution from the lowest energy pair of 
quasiparticle bands; far from the vortex core the low energy 'bound-state' bands contribute little to the order parameter. The non-monotonic behavior of the order parameter as a function of $r$ seen in Fig. 2 is due to the 'shell structure' of the quasi-bound states.

When $n_{\mu}$ is close to an integer and $\Delta$ is small the $\mathrm{BdG}$ equations can be truncated to the Landau level closest to the Fermi level. The lowest energy quasiparticle band dispersion in this quantum limit is given by: 1720

$$
E(\vec{k})=\sqrt{\xi_{N}^{2}+\left|F_{\vec{k} N N}\right|^{2}}
$$

Since $F_{\vec{k} N N}$ generically vanishes at some value of $\vec{k}$ this implies gapless behavior in the quantum limit in our two-dimensional model when $\xi_{N}=0$. For the hexagonal lattice this approximation leads to a third order zero in the excitation spectrum at the $\Gamma$ point and a first order zero at the $\mathrm{K}$ point, as pointed out by Dukan et al.19 We also find five other points along the three symmetry lines of the zone where the gap goes to zero. For threedimensional models $19 \xi_{N}$ depends on $k_{z}$ and there will always be a $k_{z}$ for which $\xi_{N}$ vanishes for each occupied Landau level in this approximation. As pointed out recently by Dukan et $a \sqrt{19}$ gapless behavior is not ruined by the weak Landau level mixing which is always present and persists for a finite range of magnetic field below $H_{c 2}$. In Fig. 7 we plot the lowest two quasiparticle energies at 21 inequivalent $\vec{k}$ points as a function of $n_{\mu}$. Near the quantum limit, gapless excitations occur for integer values of $n_{\mu}$. At weaker fields gapless excitations occur in our two-dimensional model at more widely spaced and, in general, non-integral values of $n_{\mu}$. (Presumably for a three-dimensional model, there would still be a discrete set of $k_{z}$ values at which gapless excitations occur in this regime.) The eigenvalue spectrum in the quantum limit (i.e., diagonal) approximation differs significantly from what the full theory gives and overemphasizes gapless behavior. (It is the terms off-diagonal in Landau level index which are responsible for the decrease of $T_{c 2}$ with increasing field, so one can never ignore them in the semiclassical regime.) Nevertheless, true gapped behavior sets in only when the superconducting order is sufficiently strong to increase the energies of the quasi-bound states in the vortices above $\hbar \omega_{c}$. We have been unable to divine any simple principles behind 
the seemingly complex pattern of eigenvalues in the crossover region between the quantum limit regime and the vortex core bound state regime. It appears that some kind of nonmonotonic and possibly oscillatory behavior occurs which does not originate from Landau quantization. This behavior is characterized by oscillations between wide and narrow bands, with the lowest energy state of the wide bands tending to alternate between the $\Gamma$ and $K$ points of the zone. This behavior is reminiscent of a tight-binding effect, indicating that the source of this 'long period' behavior is vortex-vortex interactions (i.e., due to the spatial modulation of the order parameter). At the $\Gamma$ point there is a pattern of oscillations which are approximately periodic in $n_{\mu}$ with a period roughly of six which reflect the fact that at this high symmetry point in the irreducible triangle only Landau levels with indices differing by six are mixed in the $u$ amplitude of the quasiparticle state. The $v$ amplitude behaves in the same way with Landau level indices which for one member of the pair of bands is offset by 1 and the other member by 3 from the $u$ amplitude Landau level indices. The field dependencies at lower symmetry $\vec{k}$ points are less simple in this regime and there is a complicated pattern of avoided level crossings. We have examined this regime for several different models and have not succeeded in identifying universal features in the electronic structure in the crossover regime except as mentioned above. In Fig. 8 we show results for the field dependence of the quasiparticle spectrum for the model with $\lambda=0.55$. For this case, one crosses over from the quantum regime to the semiclassical regime for $n_{\mu} \sim 21$. No apparent long period structure is seen in the crossover regime. For this model quasi-bound states just begin to emerge as we reach our code's limitations at $n_{\mu} \sim 40$.

In Fig. 9 we show a plot of the Landau level occupation numbers in the semiclassical regime at $n_{\mu}=40$. In the zero-field BCS case, it is known that the momentum distribution function at $\mathrm{T}=0$ is approximately that of a Fermi function at $T_{c} .30$ The analogous statement applies in the semiclassical mixed-state regime, as demonstrated in Fig. 9. A least squares fit of the Landau level occupation numbers to a Fermi function parameterized by a temperature $T$ gives an optimal $T$ equal to $T_{c 2}$ to within a few percent. 


\section{DE HAAS-VAN ALPHEN OSCILLATIONS}

To determine the magnetization $\mathrm{M}$ in the grand-canonical ensemble we self-consistently solve the mean-field equations at a set of fields spaced so that $\Delta n_{\mu}=0.1$ and evaluate the free energy using Eq. [12]. A finite difference approximation is used for the derivative with respect to field which yields a two-dimensional magnetization we define by

$$
M_{2 D}(B)=-\frac{\partial F}{\partial B}
$$

For the normal state the resulting magnetization accurately reproduces the analytically known $T=0$ result in which $M_{2 D} /\left(N \mu_{B}\right)$ is periodic in $n_{\mu}$ with period one and varies between -1 and 1 . The magnetization in the normal state jumps by $2 N \mu_{B}$ whenever the chemical potential passes through one of the degenerate Landau levels. It is the bunching of the density of states into Landau levels which leads to the period one oscillations, known as dHvA oscillations. We remark that in evaluating the magnetization we have ignored the screening of the external magnetic field so that our approach is valid only for strong type-II superconductors. For a three-dimensional system composed of isolated layers, the bulk magnetization is related to the two-dimensional magnetization defined above by $M=$ $M_{2 D} / A d$ where $A$ is the area of the two-dimensional system and $d$ is the separation between two-dimensional layers. Our approximation is valid (using Gaussian units) as long as $M<<$ $B$.

In Fig. 10 we show a plot of $M_{2 D}(H)$ for the $\lambda=0.75$ model at a very low temperature. At small $n_{\mu}$ (strong field), there are dHvA oscillations with period one as in the normal state. The dHvA oscillations are rapidly damped as the superconducting order develops. Comparing with Fig. 7 we see that strong dHvA oscillations are present only in the quantum regime where the quasiparticle bands retain a clear Landau level structure. There is some structure in the magnetization curve in the complicated crossover regime discussed above but these features are not dHvA oscillations and we suspect that they will tend to be washed out in three-dimensional models. At weaker fields $\left(n_{\mu}>\sim 22\right)$ we cross over to the 
regime where bound states are supported by the vortex core and the field dependence of the magnetization becomes relatively featureless. The negative sign of the magnetization in this region occurs because the condensation energy associated with superconducting order decreases with magnetic field. If the field dependence of these magnetization curves is Fourier transformed with respect to $n_{\mu} \propto 1 / H$, the peak at period one comes almost entirely from the quantum regime. At higher temperatures where the magnetic oscillations are damped out, we find that the magnetization is proportional to $1 / H-1 / H_{c 2}$ in contrast to GinzburgLandau theory where it is proportional to $H-H_{c 2}$.

In Fig. 11, we plot the contributions to $M_{2 D}$ from the kinetic energy and the pairing contributions to the magnetization at zero temperature. We see that in all regimes the two contributions to the magnetization oppose each other. This should be expected since the superconducting state is always formed at a cost in kinetic energy in order to take advantage of the attractive interparticle interactions. This occurs in both quantum and semiclassical limits. The quantum limit is particularly simple. Pairing is strongest when $n_{\mu}$ is an integer leading to a maximum in both the increase of kinetic energy due to superconducting order and in the pairing self-energy. The total free-energy of the system is decreased by much less than either component is shifted. The total free-energy in the normal state is at a maximum when $n_{\mu}$ is an integer and at a minimum when $n_{\mu}$ is at a midpoint between integers; at such values of $n_{\mu}$ the free energy is identical to the free energy in the absence of a magnetic field. The difference between minimum and maximum free energies is reduced by introducing superconducting order so that the amplitude of the dHvA oscillations is reduced because of superconductivity. At the same hand the amplitude of the oscillations in the kinetic energy alone is increased. This behavior reveals the importance of determining the superconducting order self-consistently within each dHvA oscillation period. Earlier work which neglected dHvA oscillations in the superconducting order itself will not be valid in general. Because of self-consistency, oscillations in the superconducting condensation energy also tend to oppose oscillations in the normal state energy. Well outside of the quantum regime this cancellation is nearly complete. Therefore the interesting work of 
Maniv et $a \sqrt{16}$ which focused on oscillations in the condensation energy alone also has limited validity. We comment further on self-consistency and the cancellation between normal state and condensation energy magnetic oscillations in the following section.

In Fig. 12, we show $M_{2 D}(H)$ for the model with $\lambda=0.55$. Again, magnetic oscillations are damped in the quantum regime and exhibit a complicated pattern in the crossover regime which is associated with the appearance and disappearance of gapless behavior as a function of field. As stated previously we believe that these oscillations will wash out for three-dimensional models. There is no simple relationship between the magnetizations in the crossover regime for the $\lambda=0.55$ and $\lambda=0.75$ models. For the $\lambda=0.55$ model, the regime of quasi-bound vortex states is just approached at the weakest magnetic fields we have considered.

Our results for these models cannot be compared directly with experiments on specific materials. However it is possible to make some general qualitative remarks that we believe are important for the interpretation of experiments. In the Lifshitz-Kosevich theory 35 of dHvA oscillations in the normal state the amplitude of the oscillations is reduced by a factor of

$$
R_{0}=\exp \left(-\pi / \omega_{c} \tau_{0}\right)
$$

because of disorder broadening of Landau levels. This specific result is based on the assumption of a magnetic field independent Lorentzian lineshape, with a full width at half maximum of $\hbar / \tau_{0}$, for the density of states of the disorder broadened Landau level. In the superconducting state (with no disorder) the density of states of a broadened Landau level is not Lorentian and, more importantly, it is not independent of magnetic field. Within each period of the dHvA oscillation the superconducting order is strongest and the Landau level is broadest when $n_{\mu}$ is an integer. This Landau level broadening reduces the increase in free-energy due to Landau quantization and therefore reduces the amplitude of the dHvA oscillations. Following Springford's group 6 : 7 we therefore assume that the damping of dHvA oscillations due to superconductivity in the mixed state is given approximately by 


$$
R_{S}=\exp \left(-\pi / \omega_{c} \tau_{S}\right)
$$

where $\hbar / \tau_{S}$ is some measure of the Landau level width in the mixed state of the $n_{\mu}$ 'th Landau level when $\xi_{n_{\mu}}=0$. (In the case of three-dimensional models, dHvA oscillations come from $k_{z}$ values where the Landau level index at the Fermi level is a local minimum or maximum (extremal orbits); the remarks below apply as well to the three-dimensional case.)

In the region near $H_{c 2}$ where dHvA oscillations are strong we can assume that only $\Delta_{0} \neq 0$ so that from Eq. 17

$$
\hbar \tau_{S}^{-1} \propto \lambda \hbar \omega_{c} \Delta_{0} n_{\mu}^{-1 / 4}
$$

The factor $n_{\mu}^{-1 / 4}$ which appears in Eq. 21] comes from the large quantum number limit 36 of the factor $D_{0}^{n_{\mu} n_{\mu}}$ in the expression for the off-diagonal self-energy; $\left(D_{0}^{n_{\mu} n_{\mu}}\right)^{2}$ is the probability 36 for two electrons with Landau level index $n_{\mu}$ to have center-of-mass kinetic energy $\hbar \omega_{c} / 2$ (Abrikosov solution) and its rate of decrease with $n_{\mu}$ in the large quantum number limit can be understood from simple phase-space considerations. The actual dispersion relation within the quasiparticle Landau band is complicated for large Landau indices and we estimate the proportionality constant in Eq. 21] by numerically calculating the standard deviation of quasiparticle energies within the band. Results are shown in Fig. 13 from which we infer that

$$
\hbar \tau_{S}^{-1} \sim 0.15 \lambda \hbar \omega_{c} \Delta_{0} n_{\mu}^{-1 / 4}
$$

As emphasized above $\Delta_{0}$ is itself a strongly oscillating function in the quantum limit so that some caution must be used in applying this relationship. An exception occurs for dHvA oscillations coming from a small piece of Fermi surface in a system where the field dependence of $\Delta_{0}$ is dominated by larger pieces of Fermi surface. In this case it may be possible to use the dHvA oscillations from a small piece of Fermi surface as a probe to look at the field dependence of $\Delta_{0}$ as suggested recently by Harrison et al.

We can use these results to estimate the typical range of fields over which dHvA oscillations from large pieces of Fermi surface will be observable. As mentioned in Sec. III 
the spatial average of the off-diagonal self-energy $F \sim 0.44 \lambda \hbar \omega_{c} \Delta_{0}$ retains its familiar BCS relationship to the critical temperature for almost all fields. Assuming this relationship, we obtain

$$
\hbar \tau_{S}^{-1} \sim 0.6 n_{\mu}^{-1 / 4} k_{B} T_{c 2}
$$

Using a Ginzburg-Landau form for the field dependence of $T_{c 2}{ }^{29}$ this becomes

$$
\hbar \tau_{S}^{-1} \sim 0.6 n_{\mu}^{-1 / 4} k_{B} T_{c} \sqrt{1-H / H_{c 2}} .
$$

dHvA oscillations will be strongly damped when the right hand side of Eq.24] is larger than $\hbar \omega_{c}$. Since the typical value of $\hbar \omega_{c}$ at $H_{c 2}$ is 3 ] $\sim\left(k_{B} T_{c}\right)^{2} / \mu$ this occurs for $\left(H_{c 2}-H\right) / H_{c 2}>$ $k_{B} T_{c} / \mu$ which is a number much less than one. (We have used $n_{\mu} \sim \mu /\left(\hbar \omega_{c}\right)$ to obtain this estimate.) For our simple model $k_{B} T_{c} / \mu \sim 0.1$ so that this simple estimate is consistent with the range of fields over which we see $\mathrm{dHvA}$ oscillations persist. For more realistic models, $k_{B} T_{c} / \mu$ is typically much smaller, at least for the largest pieces of Fermi surface, and dHvA oscillations should be seen only close to $H_{c 2}$. We remark that Eq.[24] implies that small pieces of the Fermi surface are more damped than large ones due to the $n_{\mu}^{-1 / 4}$ factor. Finally, we note that magnetic oscillations of the gap function are determined by the large pieces of the Fermi surface, and so the gap function will oscillate many times over the period of a small orbit. This could potentially complicate the analysis of such orbits.

Our results differ qualitatively from those of Maki23 and Stephen 24 who find, in our notation, that $\hbar \tau_{S}^{-1} \sim\left|F_{N N}\right|^{2} /\left(\hbar \omega_{c}\right)$ rather than $\sim\left|F_{N N}\right|$. In calculating the Landau level width these authors do not take account of the Landau level quantization of the density of final states into which the quasiparticles can be scattered. The Landau level width can then be calculated treating the scattering of quasiparticles by the vortex lattice using what is essentially a golden rule. In this language we find that the density of states in the quasiparticle Landau level is enhanced over the zero-field value by a factor of $\omega_{c} \tau_{S}$. The density of final states in the golden rule calculation is inversely proportional to $\hbar \tau_{S}^{-1}$ so that we find $\hbar \tau_{S}^{-1} \sim\left|F_{N N}\right|^{2} / \hbar \tau_{S}^{-1}$. When the Landau level is already broad because 
of disorder scattering $\left(\omega_{c} \tau_{0}<<1\right)$, which we have neglected here, the results of Maki23 and Stephen 24 are more appropriate than ours. However, in this case dHvA oscillations will be difficult to observe even in the normal state and we believe that our result will typically be more useful in analyzing experimental results. This discussion underscores the serious approximations involved in assuming that disorder and superconducting order are responsible for additive contributions to the Landau level width and correspondingly to independent attenuation factors in the expression for dHvA oscillation amplitudes. We have focused here on the situation where the dominant attenuation factor is that due to the presence of superconducting order since it is in this situation that dHvA oscillations have the greatest potential as a probe of the mixed state.

As a further test of these ideas, we have analyzed the Fourier transform of our calculations in more detail. The problem with analyzing the magnetization presented in Figs. 10 and 12 is that single period oscillations exist only over a small field range due to the rapid growth of the order parameter below $H_{c 2}$ and the limited number of Landau levels we can treat in a fully quantum approach. To circumvent this, we evaluated $M_{2 D}(H)$ at fixed $\Delta_{0}$ so as to access a large range of $n_{\mu}$ with the same input order parameter; this could be physically sensible if the superconducting order were determined primarily by pieces of Fermi surface much larger than the one being studied, which is the case for experiments done to date. These calculations were performed using the $\lambda=1$ model so that the average off-diagonal self-energy in $\hbar \omega_{c}$ units is $F=0.436 \Delta_{0}$ as discussed previously. Using the BCS ratio of $F$ to $k_{B} T_{c 2}$ of 1.76 , this would mean that $\Delta_{0} \sim 4$ should give an amplitude suppression similar to that in the normal state at $k_{B} T=\hbar \omega_{c}$. We find that as $\Delta_{0}$ is increased from zero to four, peaks in the Fourier transform of $M(H)$ at all dHvA harmonics are suppressed, and for values larger than four, no detectable peaks occur. (With our convention the fundamental harmonic of the normal state oscillations occurs in the sine transform; in our calculations, no signficant effects occur in the cosine transform except for the constant diamagnetic response). In Fig. 14, we plot the peak height of the fundamental harmonic versus $\Delta_{0}$ and compare it with the result obtained neglecting Landau level mixing and with the normal state oscillations 
at the estimated $T_{c 2} \sim 0.248 \hbar \omega_{c} \Delta_{0}$. We see that the suppression of the amplitude of the oscillations is underestimated by the diagonal approximation at all values of $\Delta_{0}$. We also find that Eq.22 provides a perfect fit to the diagonal approximation results. Comparing the initial slopes of the depression of the full non-diagonal harmonic to that of the diagonal one, we infer that the full calculation has an effective scattering rate about 3.5 times larger than the diagonal one. Given this, we can infer a scattering rate formula analogous to that of the diagonal case given in Eq.23

$$
\hbar \tau_{S}^{-1} \sim 2 n_{\mu}^{-1 / 4} k_{B} T_{c 2}
$$

The similarity of normal state oscillations at $T_{c 2}$ and the non-diagonal results also suggests that experiments might be informatively analyzed by introducing a field-dependent Dingle temperature due to the superconducting order which could be compared with $T_{c 2}(H)$. Below, we will compare both of these approaches to experimental data. Finally, we caution that the harmonic structure can be qualitatively altered by the superconducting order. This can be seen by the fact that in the non-diagonal calculation, the harmonic actually changes sign and peaks at $\Delta_{0} \sim 2$. This behavior is not a phase shift effect, rather it is due to the magnetic oscillations of the order parameter, which contribute with opposite sign to the kinetic part as in Fig. 11 (note, it is the output value of $\Delta_{0}$ from Eq. [9] which enters into the pairing energy of Eq. [13], which is why these gap oscillations contribute even at fixed input $\Delta_{0}$ ). This contribution is much weaker in the diagonal case, so no sign change occurs in that case. It is expected that self-consistency effects will wash this effect out, so it is doubtful whether such a sign change would be seen experimentally. We have found, then, that the fundamental harmonic of the non-diagonal case can be fit by a term like Eq. 22] with the same enhancement factor of 3.5 mentioned above (thus justifying Eq. 25]) plus a 'pairing' term which is proportional to $\Delta_{0}^{2}$ times a damping factor (an analogous gap oscillation term occurs in the work of Maniv et a $\sqrt{16}$ but with no damping). Similar effects happen for the higher harmonics, except that as they are suppressed more rapidly, the sign change and peak occur at smaller values of $\Delta_{0}$. This can lead to an unusual situation where the second 
harmonic can dominate the magnetization, as can be seen in Fig. 15, where we show the $M_{2 D}(H)$ data from which these Fourier transforms were evaluated for the non-diagonal case with $\Delta_{0}=1$. This highly unusual behavior might be detectable experimentally, although we caution that the strong gap oscillation contribution in the non-diagonal case will be damped additionally by self-consistent effects, so that this effect might disappear. To study these self-consistency effects in more detail will require going away from a fully quantum approach like ours to a semiclassical approximation where very large numbers of Landau levels can be treated.

With the extensive discussion above, we are now able to take a closer look at the experimental data. The most complete work to date is that of Harrison et al on $\mathrm{Nb}_{3} \mathrm{Sn} .7 \mathrm{In}$ this work, they plot the scattering rate defined in Eq. 20] versus field for three orbits on the Fermi surface. As in earlier work, these orbits are fairly small compared to the Brillouin zone dimensions. In Fig. 16, we compare the experimental scattering rate for the one orbit with the most data to three theoretical predictions (results for the other two orbits are similar). The first is the diagonal approximation of Eq.[23], the second the non-diagonal approximation of Eq. [25], and the third is extracted by equating a thermal suppression factor with a temperature equal to $T_{c 2}$ to Eq.[20]. $T_{c 2}$ was determined using the standard method of Werthamer et al. 37 As can be seen, Eq. 25] gives a very good representation of the data, with the diagonal approximation significantly underestimating the damping and a thermal suppression factor of $T_{c 2}$ strongly overestimating the damping.

\section{VARIATIONAL APPROACH}

In this section we discuss superconductivity in the mixed state from a variational point of view. In our formalism the off-diagonal self-energy (Eq.[5]) in the BdG equations is specified by a discrete set of parameters, $\Delta_{j}$. As we have mentioned previously, close to $H_{c 2}$ only $\Delta_{0}$ is significantly different from zero and the off-diagonal self-energy can then be characterized by a single parameter. Given the off-diagonal self-energy, a BCS-type variational state can 
be constructed from the eigenvectors of the $\mathrm{BdG}$ equations. We can therefore think of the $\Delta_{j}$ as a set of parameters which specify a trial wavefunction. The order parameter of this trial wavefunction is specified by a (in general) different set of parameters which are evaluated from the solution of the BdG equations using Eq. 9 ; in the following we refer to the two sets of parameters as $\Delta_{j}^{i n}$ and $\Delta_{j}^{\text {out }}$. The expectation value of the Hamiltonian in this wavefunction is given by Eq. 12] with the pairing energy evaluated from Eq. 13. using $\Delta_{j}^{\text {out }}$. It is clear from the variational derivation of the BdG equations that the free energy of the model has extrema at the $\Delta_{j}$ which are self-consistent solutions of the BdG equations.

We will discuss in detail the approximation where we consistently assume that only $\Delta_{0} \neq 0$ so that the superconducting state is characterized by a single parameter as in the zero-field situation. We note that the off-diagonal self-energy, and therefore the variational state, depend only on the combination $\lambda \Delta_{0}^{i n} \equiv \tilde{\Delta}_{0}^{i n}$. In Fig. 17 we plot the grand potential at $k_{B} T / \mu=10^{-4}(\Omega=E-\mu N)$ as a function of the variational parameter $\tilde{\Delta}_{0}^{i n}$ at $n_{\mu}=9.5$ and $n_{\mu}=10$ for $\lambda=0.75, \lambda=1.00$, and $\lambda=1.25$. In the normal state, the grand potential reaches its minimum when the chemical potential is at the mid-point between Landau levels, i.e., when $n_{\mu}$ is half an odd integer. At these chemical potential values the normal state grand potential equals its zero-field value $\Omega_{0}$. The grand potential has a local maximum when $n_{\mu}$ is an integer; at integral values of $n_{\mu}$ the relative increase in the grand potential compared to its zero-field value is $1 /\left(2 n_{\mu}+1\right)^{2}$. In Fig. 17 we see that for $\lambda=0.75$ the free energy minimum occurs at $\tilde{\Delta}_{0}^{i n}=0$ for $n_{\mu}=9.5$ and at $\tilde{\Delta}_{0}^{i n} \sim 1.8$ for $n_{\mu}=10$. Integral values of $n_{\mu}$ are more favorable for superconductivity because of the high density of states at the Fermi level. The small condensation energy at $n_{\mu}=10.0$ reduces the grand potential difference between the two fields and therefore reduces the amplitude of the dHvA oscillations. This is an alternate point of view on the damping of dHvA oscillations in the quantum regime. For the larger values of $\lambda$ the superconducting condensation energy is either comparable to or larger than the amplitude of the magnetic oscillations in the normal state grand potential. For $\lambda=1.25$ the minimum grand potential occurs in the vortex core bound state regime where magnetic oscillations are essentially absent. In this regime the 
grand potential is lower at larger $n_{\mu}$ (weaker field) as expected because of the diamagnetism of the mixed state, but the magnetic field dependence is not periodic in $n_{\mu}$.

Using this approach we can study the dependence of the equilibrium order parameter on the model parameter $\lambda$. From the self-consistency condition we see that the value of $\lambda$ at which the self-consistent order parameter value is $\Delta^{\text {out }}$ is

$$
\lambda=\frac{\tilde{\Delta}_{0}^{i n}}{\Delta_{0}^{o u t}\left[\tilde{\Delta}_{0}^{i n}\right]} .
$$

In Fig. 18 we plot the order parameter as a function of $\lambda$ for our model at $n_{\mu}=40$ and $n_{\mu}=30$ at $k_{B} T / \mu=10^{-2}$. For $n_{\mu}=40$ results are also shown for $k_{B} T / \mu=10^{-4}$. We note that the order parameter grows like $\left(\lambda-\lambda_{c}\right)^{1 / 2}$ for $\lambda>\lambda_{c}$ where $\lambda_{c}$ is the coupling strength required for superconductivity to occur. This is the expected result within meanfield theory. $\lambda_{c}$ decreases with decreasing magnetic field and is expected to approach zero at zero field since in that limit superconductivity occurs in this model at arbitrarily small $\lambda$. For $k_{B} T / \mu=10^{-4}$ the inequality $k_{B} T<<\hbar \omega_{c}$ is satisfied and the quantum regime of strong magnetic field superconductivity is seen in the persistence of superconducting order to very small $\lambda$ values. At half odd integral values of $n_{\mu}$ the order parameter curve would be shifted toward larger values of $\lambda$ rather than smaller values of $\lambda$ in the quantum regime.

In Fig. 19 we compare the dependence of the superconducting condensation energy $F_{c}$ on the order parameter with expectations based on Ginzburg-Landau theory by plotting $F_{c} /\left(\Delta_{0}^{\text {out }}\right)^{2}$ against $\left(\Delta_{0}^{\text {out }}\right)^{2}$. (We define $F_{c}$ in the grand canonical ensemble as the difference between the normal state grand potential and the superconducting state grand potential.) These results are for $\lambda=1$; with our simple model results at other values of $\lambda$ differ only by a constant vertical shift of the curves. Plots are shown for $k_{B} T / \mu=10^{-2}$ at $n_{\mu}=40$ and $n_{\mu}=30$ and for $k_{B} T / \mu=10^{-4}$ at $n_{\mu}=40$ as in Fig. 18. In Ginzburg-Landau theory 30 these plots should give straight lines whose $y$ intercepts are proportional to the coefficient of the quadratic term in the Ginzburg-Landau energy functional for the lowest Landau level of the Cooper pairs and whose slopes are proportional to the coefficient of the quartic term in the Ginzburg-Landau energy functional. The intercept is expected to increase with increasing 
magnetic field and with increasing temperature in agreement with our calculations. We see evidence in Fig. 19 for some temperature and magnetic field dependence in the coefficient of the quartic term which is expected far away from the zero-field critical temperature but is normally neglected in Ginzburg-Landau theory. A large departure from GinzburgLandau theory is seen at small order parameters for the low temperature case. Again this deviation appears only in the quantum regime where pairing is dominantly within an individual Landau level. At half odd integral values of $n_{\mu}$ the magnitude of the condensation energy would be decreased rather than increased upon entering the quantum regime.

\section{SUMMARY}

In this paper, we have presented an exact mean field treatment of the flux lattice state and discussed in detail the resulting quasiparticle electronic structure and magnetization in the 2D limit. We have found that as the field is lowered, the Landau levels nearest the chemical potential cross over to vortex core bound states. Moreover, the field dependence of the quasiparticle electronic structure shows rich behavior, due to the strong field dependence of the Landau levels, order parameter, and vortex-vortex interactions, and their interplay with one another. We also find that as the field is lowered below $H_{c 2}$, the single period magnetic oscillations become rapidly damped due to broadening of the Landau levels, and essentially disappear when the average superconducting gap becomes of order the cyclotron energy. This is consistent with observations of the dHvA effect in the mixed state. For lower fields, gaplessness can still occur, but the uniform periodicity of the gaplessness (as occurs near $\left.H_{c 2}\right)$ is destroyed by the various competing effects mentioned above. For even lower fields, the vortex core regime is reached and gaplessness no longer occurs, but there is still structure in the magnetization driven by the spatial modulation of the order parameter. We would like to conclude by saying that the rich behavior we predict for the field dependent quasiparticle electronic structure has important implications not only for the dHvA effect, but for all experiments which measure low energy excitations in the vortex lattice state. 


\section{ACKNOWLEDGMENTS}

This work was supported in part by the U.S. Dept. of Energy, Basic Energy Sciences, under Contract No. W-31-109-ENG-38, and in part by the National Science Foundation under grant DMR-9416906. The authors would like to thank Prof. Mike Springford for sending us a preprint of the work of his group on $\mathrm{Nb}_{3} \mathrm{Sn}$ and Profs. Steven Hayden and Michael Naughton for discussions concerning experimental data. M.R.N. would like to acknowledge

the hospitality of the Cavendish Laboratory, Cambridge University, where some of this work was begun. 


\section{REFERENCES}

${ }^{1}$ See for example D. Shoenberg, Magnetic oscillations in metals (Cambridge University Press, 1984).

${ }^{2}$ A.A. Abrikosov, Zh. Eksp. Teor. Fiz. 32, 1442 (1957).

${ }^{3}$ J.E. Graebner and M. Robbins, Phys. Rev. Lett. 36, 422 (1976).

${ }^{4}$ Y. Onuki et al, J. Phys. Soc. Japan 61, 692 (1992); R. Corcoran et al, J. Phys. Cond. Mat. 6, 4479 (1994).

${ }^{5}$ F.M. Mueller, D.H. Lowndes, Y.K. Chang, A.J. Arko, and R.S. List, Phys. Rev. Lett. 68, 3928 (1992).

${ }^{6}$ R. Corcoran, N. Harrison, S.M. Hayden, P. Meeson, M. Springford, and P.J. van der Wel, Phys. Rev. Lett. 72, 701 (1994).

${ }^{7}$ N. Harrison, S.M. Hayden, P. Meeson, M. Springford, P.J. van der Wel, and A.A. Menovsky, Phys. Rev. B 50, 4208 (1994).

${ }^{8}$ C.M. Fowler, B.L. Freeman, W.L. Hults, J.C. King, F.M. Mueller, and J.L. Smith, Phys. Rev. Lett. 68, 534 (1992); F.M. Mueller, J. Phys. Chem Solids 52, 1457 (1991) and 53, 1549 (1992).

${ }^{9}$ G. Kido, K. Komorita, H. Katayama-Yoshida, and T. Takahashi, J. Phys. Chem. Solids 52, 1465 (1991) and 53, 1555 (1992).

${ }^{10}$ E.G. Haanappel et al, J. Phys. Chem. Solids 54, 1261 (1993).

${ }^{11}$ R.G. Goodrich et al, J. Phys. Chem. Solids 54, 1251 (1993).

${ }^{12}$ A.K. Rajagopal and R. Vasudevan, Phys. Lett. 23, 539 (1966); L.W. Gruenberg and L. Gunther, Phys. Rev. 176, 606 (1968).

${ }^{13}$ For recent results on the linearized gap equations which determine $H_{c 2}$ see A.K. Rajagopal 
and J.C. Ryan, Phys. Rev. B 44, 10280 (1991); A.H. MacDonald, H. Akera, and M.R. Norman, Phys. Rev. B 45, 10147 (1992).

${ }^{14}$ Z. Tesanovic and M. Rasolt, Phys. Rev. B 39, 2718 (1989); Z. Tesanovic, M. Rasolt, and L. Xing, Phys. Rev. Lett. 63, 2425 (1989) and Phys. Rev. B 43, 288 (1991).

${ }^{15}$ M.R. Norman, H. Akera, and A.H. MacDonald, in Physical Phenomena at High Magnetic Fields, eds. E. Manousakis, P. Schlottmann, P. Kumar, K. Bedell, and F.M. Mueller (Addison-Wesley, Reading, 1992), p. 326.

${ }^{16}$ T. Maniv, A.I. Rom, I.D. Vagner, and P. Wyder, Phys. Rev. B 46, 8360 (1992); T. Maniv, I.D. Vagner, and P. Wyder, J. Phys. Chem. Solids 54, 1283 (1993).

${ }^{17}$ H. Akera, A.H. MacDonald, S.M. Girvin, and M.R. Norman, Phys. Rev. Lett. 67, 2375 (1991).

${ }^{18}$ V.N. Nicopoulos and P. Kumar, Phys. Rev. B 44, 12080 (1991).

${ }^{19}$ S. Dukan, A.V. Andreev, and Z. Tesanovic, Physica C 183, 355 (1991); S. Dukan and Z. Tesanovic, Phys. Rev. B 49, 13017 (1994).

${ }^{20}$ M.J. Stephen, Phys. Rev. B 43, 1212 (1991).

${ }^{21}$ J.C. Ryan and A.K. Rajagopal, Phys. Rev. B 47, 8843 (1993).

${ }^{22}$ M. Rasolt, Phys. Rev. Lett. 58, 1482 (1987); M. Rasolt and Z. Tesanovic, Rev. Mod. Phys. 64, 709 (1992).

${ }^{23}$ K. Maki, Phys. Rev. B 44, 2861 (1991).

${ }^{24}$ M.J. Stephen, Phys. Rev. B 45, 5481 (1992).

${ }^{25}$ U. Brandt, W. Pesch, and L. Tewordt, Z. Phys. 201, 209 (1967); A. Houghton and K. Maki, Phys. Rev. B 4, 843 (1971); K. Maki and A. Houghton, Phys. Rev. B 4, 847 (1971).

${ }^{26}$ M.R. Norman, H. Akera, and A.H. MacDonald, Physica C 196, 43 (1992). 
${ }^{27}$ A.K. Rajagopal, Phys. Rev. B 46, 1224 (1992).

${ }^{28}$ H. Akera, A.H. MacDonald, and M.R. Norman, Physica B 184, 337 (1993).

${ }^{29}$ See for example, P.G. de Gennes, Superconductivity in Metals and Alloys (Addison-Wesley, Reading, 1989) Chapter V.

${ }^{30}$ See for example, M. Tinkham, Introduction to Superconductivity (Krieger, 1985).

${ }^{31}$ R.S. Markiewicz, I.D. Vagner, P. Wyder, and T. Maniv, Solid State Comm. 67, 43 (1988).

${ }^{32}$ C. Sa de Melo, unpublished.

${ }^{33}$ D. Shoenberg, J. Low Temp. Phys. 56, 417 (1984).

${ }^{34}$ C. Caroli, P.G. de Gennes, and J. Matricon, Phys. Lett. 9, 307 (1964); E.B. Hansen, Phys. Lett. 27A, 576 (1968); J. Bardeen, R. Kummel, A.E. Jacobs, and L. Tewordt, Phys. Rev. 187, 556 (1969); W. Bergk and L. Tewordt, Z. Phys. 230, 178 (1970).

${ }^{35}$ I.M. Lifshitz and A.M. Kosevich, Zh. Eksp. Teor. Fiz. 29, 730 (1955); A.A. Abrikosov, Fundamentals of the Theory of Metals (North-Holland, Amsterdam, 1988), Chap. 10.

${ }^{36}$ A.H. MacDonald, H. Akera, and M.R. Norman, Aust. J. Phys. 46, 333 (1993).

37 N.R. Werthamer, E. Helfand, and P.C. Hohenberg, Phys. Rev. 147, 295 (1966). 


\section{FIGURES}

FIG. 1. $T_{c 2}$ and the spatial average of the off-diagonal self-energy $F$ at $k_{B} T / \mu=10^{-3}$ in units of the chemical potential, $\mu$, versus $n_{\mu}$. These results are for the $\lambda=0.75$ model with a smooth cut-off as discussed in the text. All results are for this model unless otherwise noted.

FIG. 2. Magnitude of the order parameter $(\Delta)$ at $k_{B} T / \mu=10^{-3}$ along a line between neighboring vortices on the triangular vortex lattice. $r$ is the distance from one vortex in units of the near-neighbor distance. The ten curves are for $n_{\mu}=12,15, \cdots, 39$. The order parameter is calculated in units of $\left(4 \pi \ell^{2}\right)^{-1}$. In these units the off-diagonal self-energy is obtained by multiplying by $\frac{\lambda}{2} \hbar \omega_{c}$. As the field is lowered ( $n_{\mu}$ is increased ) the order parameter becomes larger and the ratio of the vortex core size to the period of the vortex lattice becomes smaller.

FIG. 3. Lowest ten quasiparticle energies along the perimeter of the irreducible triangle in $\vec{k}$-space for $n_{\mu}=15$. The energies are expressed in units of $\hbar \omega_{c}$.

FIG. 4. As in Fig.3 but for $n_{\mu}=40$.

FIG. 5. Magnetic field dependence of the average quasiparticle energies for the lowest five pairs of bands. The field is parameterized by $n_{\mu}$ and the quasiparticle energies are expressed in terms of $\mu$.

FIG. 6. Contributions to the order parameter from the four lowest energy quasiparticle bands. The modulus of the order parameter is plotted along the same line connecting neighboring vortices as in Fig. 2. These results are for $n_{\mu}=40$. To aid clarity we plot the modulus of the contributions from the first two bands and the negative of the modulus of the contributions from the second two bands. We see that the contributions from each member of a pair are essentially identical. The contribution of the first pair of quasiparticle bands corresponds to what is expected for the lowest energy bound state in an isolated vortex core while the contribution from the second pair of bands corresponds to what is expected from the next bound state in an isolated vortex core. 
FIG. 7. Magnetic field dependence of quasiparticle energies at 21 inequivalent $\vec{k}$ points in the irreducible triangle for the four lowest bands. The quasiparticle energies are in units of $\hbar \omega_{c}$ and the field is parameterized by $n_{\mu}$. In these field-dependent units the energy of field-independent eigenvalues is proportional to $n_{\mu}$. We see the emergence of weakly field dependent quasi-bound states at low fields.

FIG. 8. As in Fig.7 but for the model with $\lambda=0.55$.

FIG. 9. Landau level occupations numbers in the mixed state at $k_{B} T / \mu=10^{-3}$ for $n_{\mu}=40$. The solid curve is the Fermi distribution function evaluated at $T=T_{c 2}$ at the same magnetic field.

FIG. 10. $M_{2 D}(H)$ for the $\lambda=0.75$ model versus $n_{\mu}$. The magnetization per particle is in units of $\frac{e \hbar \mu}{m^{*} c}$.

FIG. 11. Decomposition of $M_{2 D}(H)$ into kinetic and pairing energy contributions for the $\lambda=0.75$ model.

FIG. 12. $M_{2 D}(H)$ versus $n_{\mu}$ for the $\lambda=0.55$ model.

FIG. 13. Quasiparticle Landau level width (standard deviation for $66 \vec{k}$ points) at $\xi_{n_{\mu}}=0$ $\left(\hbar \tau_{S}^{-1}\right)$ at integral $n_{\mu}$ neglecting inter-Landau level pairing for an input $\Delta_{0}=1$ (results including inter-Landau level terms are very similar). In this approximation, the band width is proportional to $\Delta_{0}$. The curve is a fit to a power law of $n_{\mu}^{-1 / 4}$.

FIG. 14. Plot of the fundamental harmonic of the sine Fourier transform of $M_{2 D}(H)$ over the interval from $n_{\mu}=20$ to $n_{\mu}=40$ as a function of input $\Delta_{0}$ (solid circles). The solid diamonds show results obtained neglecting Landau level mixing and the open circles are for the normal state with a temperature equal to $0.248 \hbar \omega_{c} \Delta_{0}$, the estimated $T_{c 2}$. For these calculations, $\lambda=1$ and $k_{B} T / \mu=10^{-4}$. 
FIG. 15. $M_{2 D}(H)$ for an input $\Delta_{0}=1$ (solid line) in the calculations of Fig.14. The dashed lines show results obtained when Landau level mixing is neglected.

FIG. 16. $\tau_{S}^{-1}$ in TeraHertz units versus field in Tesla extracted from the dHvA data of Ref. 7 for the 581 Tesla orbit of $\mathrm{Nb}_{3} \mathrm{Sn}$ (solid circles) compared to results of the diagonal approximation of Eq.[23] (+), including inter-Landau level effects, Eq.[25] (x), and to a thermal damping factor equal to $T_{c 2}$ (open circles). Results for other orbits are similar. $H_{c 2}$ is 19.7 Tesla.

FIG. 17. Dependence of the grand potential on the variational parameter $\tilde{\Delta}_{0}^{i n}$ for three different values of $\lambda$ with $k_{B} T / \mu=10^{-4}$. The dashed curves are for $n_{\mu}=10$ and the solid curves are for $n_{\mu}=9.5$

FIG. 18. Equilibrium order parameter as a function of $\lambda$. The solid line is for $n_{\mu}=30$ and $k_{B} T / \mu=10^{-2}$; the dashed line is for $n_{\mu}=40$ and $k_{B} T / \mu=10^{-2}$; the long-dashed line is for $n_{\mu}=40$ and $k_{B} T / \mu=10^{-4}$.

FIG. 19. Superconducting condensation energy as a function of the order parameter of the variational wavefunction, $\Delta_{0}^{\text {out }}$. The solid line is for $n_{\mu}=30$ and $k_{B} T / \mu=10^{-2}$; the dashed line is for $n_{\mu}=40$ and $k_{B} T / \mu=10^{-2}$; the long-dashed line is for $n_{\mu}=40$ and $k_{B} T / \mu=10^{-4}$. 